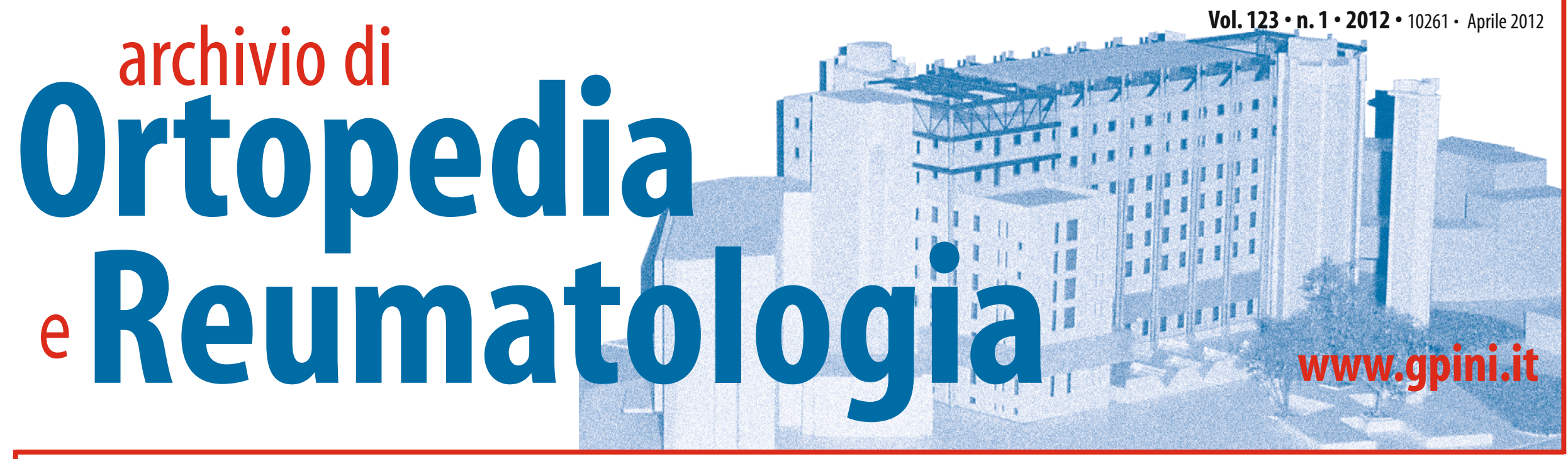

\title{
Novità in ortopedia e traumatologia pediatrica
}

a cura di Antonio Memeo

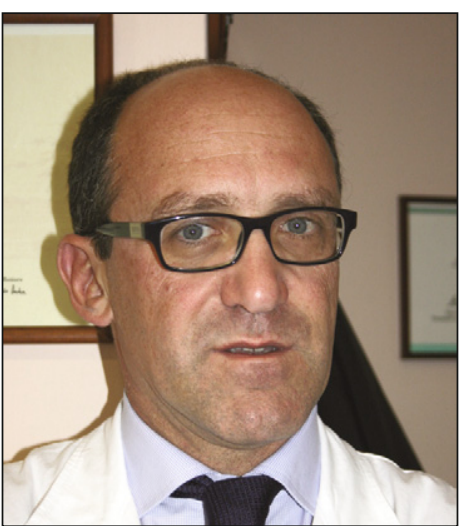

A. Memeo

Negli ultimi anni sono state molte le novità introdotte in ortopedia e traumatologia pediatrica e ho pensato che dedicare un fascicolo dell'Archivio di Ortopedia e Reumatologia fosse importante per poterle esaminare in dettaglio. Per questo motivo ho invitato a illustrarle molte personalità scientifiche che da sempre si occupano di questi problemi.

Partendo dall'epoca pre-natale e post-natale, abbiamo visto che le nuove metodiche di ecografia dinamica consentono diagnosi che sino a qualche anno fa non era possibile effettuare se non tardivamente, come nei casi di piede torto congenito, che spesso viene già diagnosticato in utero con le ecografie morfologiche, e come nella cura della displasia congenita dell'anca, dove è possibile monitorare la terapia ricorrendo sempre meno all'utilizzo della radiologia tradizionale.

E poi evidente come negli ultimi anni si sia modificato il trattamento delle fratture dei bambini, dove si cerca di ricorrere sempre meno alle immobilizzazioni in apparecchio gessato cercando invece di utilizzare tecniche chirurgiche poco invasive che consentano ai pazienti di riprendere le attività sia scolastiche sia ludiche nel più breve tempo possibile; infatti in traumatologia pediatrica si utilizzano sempre più spesso sistemi di fissazione interna ed esterna anche in pazienti al di sotto di 56 anni.

Limpiego sempre crescente dell'interventistica in traumatologia infantile permette ai bambini recuperi rapidi, consentendo loro di deambulare precocemente e di evitare quelle ipotrofie muscolari causate dagli apparecchi gessati utilizzati sino a poco tempo fa. In questo settore oggigiorno è possibile utilizzare anche tecniche biofisiche di stimolazione del callo osseo che ne consentono una formazione più rapida, con indubbi vantaggi dal punto di vista funzionale. Tali metodologie vengono impiegate nelle più varie situazioni patologiche, come i ritardi di consolidazione, le necrosi vascolari della testa femorale, le fratture vertebrali, le patologie della cartilagine, gli edemi dell'osso sottocondrale e le osteocondriti tipiche dell'adolescenza.

Queste innovazioni tecnologiche sono sempre più di ausilio nella nostra professione di ortopedic pediatrici e hanno oggi ottenuto un riconoscimento anche nella letteratura internazionale.

I mezzi di sintesi sono cambiati e attualmente si cerca di ricorrere sempre più a materiali biodegradabili i quali, una volta impiantati, danno garanzie di tenuta paragonabile a quella delle sintesi metalliche e hanno l'indubbio vantaggio di non dover essere rimossi con un secondo intervento, evitando ai bambini e alle famiglie lo stress anche emotivo di un'ulteriore ospedalizzazione. Queste sintesi si applicano non solo nelle fratture, ma possono essere utilizzate anche nella epifisiolisi per fissare la testa femorale e hanno ottenuto un largo utilizzo anche in patologie come il piede piatto, dove hanno ormai raggiunto una diffusione su larga scala.

Dopo più di un decennio questi nuovi materiali si sono dimostrai affidabili e con poche complicanze, sostituendo in molti casi le sintesi tradizionali.

Da non dimenticare la traumato- logia dello sport, che ultimamente coinvolge sempre più adolescenti ai quali vengono richieste prestazioni paragonabili a quelle degli atleti professionisti. Da no tare che i soggetti in accrescimento troppo sollecitati da microtraumi sviluppano sofferenze tendinee e muscolari che poi si ripercuotono anche sull'accrescimento dell'osso. Oggi si possono prevenire e trattare queste forme, che solo sino a pochi anni fa venivano curate esclusivamente con il riposo e l'astensione assoluta dall'attività. Anche nel campo delle ricostruzioni legamentose sono stati fatti passi avanti importanti, permet tendo di anticipare anche di molto le ricostruzioni del LCA con tecniche che non vanno a intaccare le cartilagini di accrescimento e riportano a stabilità un'articolazione che diversamente avrebbe dovuto aspettare anni per essere sistemata, con evidenti implicazioni sull'usura cartilaginea. In chirurgia ortopedica infantile non bisogna dimenticare l'apporto delle tecniche artroscopiche, che permettono di risolvere patologie una volta ritenute di difficile trattamento. Si può effettuare agevolmente la chirurgia del ginocchio anche nei piccoli pazienti affetti da menisco discoide sintomatico, da osteocondriti della cupola astragalica e del condilo femorale. È anche possibile con l'artroscopia d'anca, in casi selezionati, effettuare lavaggi articola- ri nelle artriti settiche degli adolescenti.

Le tecniche di fissazione esterna sono oggi sempre più impiegate nel nostro settore, oltre che per allungare gli arti, anche per correggere le deformità assiali e rotatorie congenite o post-traumatiche, e ultimamente anche nella cura delle rigidità del piede torto congenito. Da non dimenticare che in questa patologia oggi si sono diffuse tecniche che praticamente quasi escludono la chirurgia, riservandola ai casi che recidivano. Il metodo di Ponseti permette di trattare il PTC con gessi, tutori, allungamenti tendinei percutanei e manipolazioni.

Grossi sviluppi si sono avuti con l'avvento delle metodiche per allungare gli arti nella cura dell'acondroplasia; anche se, come ormai sostengono in molti, questa non è una malattia, ma la condizione particolare di un individuo con il gene FGFR3 diverso da quello del restante 99,996\% della popolazione, il paziente acondroplasico può oggi permettersi di avere una vita di relazione migliore, sia pure con sacrifici legati al lungo percorso chirurgico. Un ausilio particolare nel followup di questi pazienti viene dato da una équipe multispecialistica comprendente non solo i chirurghi che effettuano gli interventi, ma anche gli endocrinologi pediatrici, gli psicologi, i fisiatri e i fisioterapisti che accompagnano i bambini e i loro genitori in questa strada lunga e difficile. Nel settore della chirurgia protesica si è ormai estesa a livello nazionale la protesizzazione giovanile, che sino a pochi anni fa era un evento raro e discusso mentre al giorno d'oggi le indicazioni in tal senso sono sempre più diffuse, grazie al miglioramento dei materiali. Molte malattie tipiche dell'infanzia, come gli esiti della lussazione congenita dell'anca, gl esiti della malattia di Perthes, dell'epifisiolisi e di forme autoimmuni trattate con terapie mediche, che come effetto collaterale portano a necrosi della testa femorale, vengono sempre più spesso curate con protesi anche in pazienti al di sotto di 18 anni di età. In tal modo si permette di avere una vita di relazione normale anche ad adolescenti che diversamente avrebbero avuto problemi di inserimento scolastico e lavorativo.

I progressi nella diagnostica precoce e le terapie mediche e chirurgiche dei tumori infantili portano ormai a guarigione con pochi esiti casi che solo pochi anni fa non avrebbero potuto essere curati se non con amputazioni.

Lutilizzo della TAC dal 1974 e poi l'estensione della RMN nei primi anni Ottanta permettono di effettuare diagnosi precise e di indirizzare la programmazione chirurgica ed effettuare follow-up e

continua a pag. 2 > 


\section{Board Scientifico}

Direttori Scientifici:
M.d'Imporzano, Milano
C.Verdoia, Milano
Vice Direttori Scientifici:
M. Berruto, Milano
S. Brambilla, Milano
Direttore Responsabile:
A. Tropiano, Milano

\section{Comitato Scientifico:}

P. Bartolozzi, Verona - F. Bertoni, Bologna

F. Biggi, Belluno - R. Capanna, Firenze P. Cherubino, Varese - R.D. D'Ambrosia, New Orleans - L. Del Sasso, Como A. Diméglio, Montpellier - W.F. Enneking, Gainesville - F. Fantini, Milano - P.G. Garbagna, Milano - S. Giannini, Bologna A. Lanzetta, Milano - E. Meani, Milano F.Odella, Milano - A. Parafioriti, Milano G. Randelli, Milano - P. Rossi, Torino - C Verdoia, Milano - V.Zucchi, Milano

\section{Comitato Editoriale}

S. Caserta, Milano - R. Coluccia, Milano C. Corradini, Milano - C. Cucciniello, Milano - F. Di Domenica, Milano - G. Di Luca, Milano - M. Gallazzi, Milano - V. Gerloni, Milano - G. La Maida, Milano - G. Laurà, Milano - F. Maggi, Milano - S. Mapelli, Milano - B. Marelli, Milano - E.C. Marinoni, Milano - M. Mazza, Milano - E. Paresce, Milano - L.Sinigaglia, Milano - G. Sironi, Milano - F. Torretta, Milano - S. Tosi, Milano A. Ventura, Milano - R. Viganò, Milano

\section{Comitato di Redazione}

W.Albisetti,Milano-M.Berruto,MilanoS Brambilla, Milano - U. Dacatra, Milano L. de Girolamo, Milano - F. Fischer, Milano P.L. Gambrioli, Milano - L. Pierannunzii, Milano - L.Sinigaglia, Milano - A.Ventura, Milano - S.Zeni,Milano

\section{Segreteria di Redazione:} N.Zerbi, Milano - S.Zeni, Milano

\section{Aziende sostenitrici}

Adler Ortho, Cormano (MI)

Ceraver, Bologna

Eurosets, Medolla (MO)

IGEA, Carpi (MO)

\section{Orthopediatrics-Medical 2011, Milano}

Redax, Poggio Rusco (MN)

Sintea Plustek, Assago (MI)

Synthes, Opera (MI)

\section{Colophon}

\section{Coordinamento editoriale:} Carlotta d'Imporzano

Elena Bernacchi

Journal Department

Springer-Verlag Italia SrI

Via Decembrio 28, 20137 Milano

Tel.: 025425 9732/65

Fax:02 54259701

e-mail:

carlotta.dimporzano@springer.com

elena.bernacchi@springer.com

\section{Grafica e impaginazione:}

Graphostudio, Milano

\section{Stampa:}

Grafiche Erredue, Cirimido (CO)

Registrazione del Tribunale di Milano N. 400 del 24 ottobre 1981

Registro Nazionale della Stampa,

posizione N. 9016

La distribuzione in Italia avviene

ai sensi della legge 675/96

Springer fa parte di

Springer Science + Business Media

springer.com

\section{springer.com \\ OSpringer-Verlag Italia, 2012 \\ Via Decembrio 28, \\ 20137 - Milano, Italia \\ Stampato in Italia \\ Versione elettronica e realizzazione Web: \\ Anna Gallicchio \\ smm Srl - Scientific Multimedia \\ http://www.smm-srl.it \\ Corso Vercelli 9 - 20144 Milano \\ e-mail:a.gallicchio@smm-srl.it}

\section{Abbonamenti 2012:}

ISSN 0390-7368 (versione cartacea)

ISSN 1592-7113 (versione elettronica)

$€ 83,00$ (4 fascicoli),

inclusa la versione on-line

Gli ordini dovranno essere indirizzati Springer-Verlag Italia Sr

Journal Department

Via Decembrio 28

20137 Milano

e-mail: elena.bernacchi@springer.com

\section{Springer}

la rivista on-line

Vieni a visitarci all'indirizzo http://www.gpini.it

User ID: arch2001

Password: pini

Di prossima pubblicazione
\[ \begin{array}{l}\text { L'anestesia in ortopedia } \\ \text { Coordinatore Gabriele Cornaggia }\end{array} \]
Editoriale a cura di G. Cornaggia
Tromboprofilassi e problematiche anestesiologiche
A. Fanelli et al.
Blocchi perinervosi continui
G. Cappelleri et al.
II buon uso del sangue
P. Ghisi et al.
Anestesia in day surgery: nuove prospettive
A. Ambrosoli et al.
Ruolo dell'ecografia in ALR D
P. Ghisi, G. Danelli
Anestesia nel grande anziano: generale vs ALR
L. Pacileo et al.
Nuove tecniche di imaging (eco 3D, epiduroscopia ecc.)
P. Grossi et al.
Dolore cronico: il ruolo dell'anestesia loco-regionale
M. Allegri et al.
ALR nella chirurgia pediatrica
V. Mossetti et al.
Omeostasi termica nei pazienti sottoposti a interventi
di chirurgia ortopedica e traumatologica
A. D'Aloia et al.

\section{INDICE}

NOVITÀ IN ORTOPEDIA E TRAUMATOLOGIA PEDIATRICA

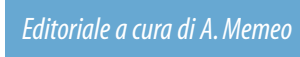

Evoluzione nelle tecniche di osteosintesi transossea in ortopedia

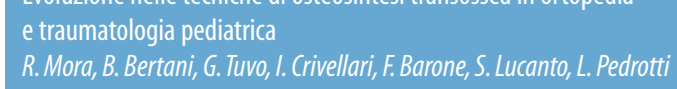

II trattamento delle cisti ossee aneurismatiche in età evolutiva R. Zorzi, P.A. Daolio, S. Bastoni, A. Parafioriti, W. Albisetti, S. Mapelli

\section{L'artroprotesi totale d'anca nel paziente giovane}

P.V. Frediani, A. Pascazio, D. Baì

Evoluzione nel trattamento delle fratture diafisarie del bambino

R. Facchini

L'artrografia nel distacco epifisario dell'omero distale nel neonato. Case report

V. Corni, C. Origo

Trattamento delle fratture diafisarie di femore e tibia in età pediatric con chiodi endomidollari elastici

A. Andreacchio, M. Paonessa, L. Marengo

\section{Strategie nel trattamento conservativo del piede torto congenito} C. Viganò, L.S. Giarratana, F. Spreafico, G. Minnici, W. Albisetti

Materiali bioriassorbibili in ortopedia e traumatologia pediatrica

O. Donzelli, L. Marchesini Reggiani, M. Lampasi, D. Antonioli

II trattamento dinamico nella displasia evolutiva dell'anca

G. Pagnotta, L. Ruzzini, L. Oggiano

II trattamento delle deviazioni assiali degli arti inferiori con fissatore esterno F. Verdoni, L. Pedretti

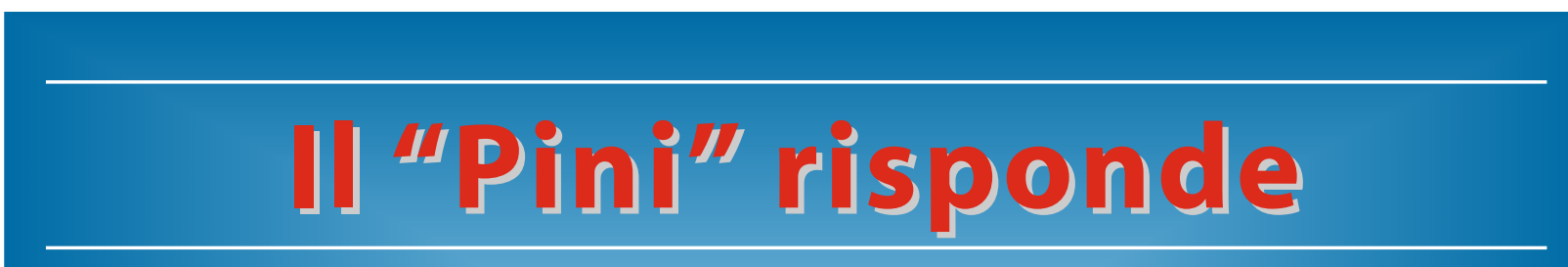

L'Istituto Ortopedico Gaetano Pini sta attivando un servizio di linea diretta fra i lettori della rivista e i medici dell'Istituto, che risponderanno a quesiti posti da colleghi su argomenti di carattere specialistico in ambito ortopedico traumatologico, riabilitativo e reumatologico.
Si prega di inviare le richieste via e-mail al seguente indirizzo:

ilpinirisponde@gpini.it

N.B. Questo servizio è dedicato esclusivamente ai Medici e Chirurghi; non verranno quindi prese in considerazione domande poste da persone che non siano medici.

stadiazioni accurate. Anche in | giovani ortopedici e cerca di da- $\mid$ che l'Istituto Gaetano Pini non questo settore, come in quello del- $\quad$ re possibilità di ricerca, anche se perdesse la sua vocazione verso la le patologie reumatiche, l'utilizzo i fondi disponibili nel nostro set- $\quad$ cura delle patologie infantili.

di nuovi farmaci antitumorali per- $\quad$ tore superspecialistico sono sem- $\quad$ Mi piacerebbe concludere questa mette di ridurre le resezioni chi- pre molto limitati.

rurgiche e di riservare solo a po- Imeriti di queste innovazioni vanchi casi interventi demolitivi. Og- no ai cultori dell'ortopedia e traugi mediante tecniche mini-invasi- $\quad$ matologia infantile che negli ultive si possono curare con ablazio- $\quad$ mi decenni si sono dedicati a svini selettive in radiofrequenza, sot- luppare ricerche in questo settore. to controllo TAC, gli osteomi Il nostro Istituto è da sempre alosteoidi che, per l'atipicità delle l'avanguardia in questo campo e loro localizzazioni, una volta ri- mi fa particolarmente piacere cichiedevano una chirurgia diffici- tare alcuni eminenti chirurghi che le e spesso molto impegnativa. hanno diretto l'Ortopedia pediaUn grande sviluppo all'ortopedia trica, come il Prof. Ruffoni, il Prof. e traumatologia degli anni 2000 lo Zaffaroni, il Prof. Dragonetti e il sta dando la nostra Società Scien- mio maestro, il Prof. Peretti, che tifica, SITOP, che promuove con- $\quad$ nell'ultimo decennio con la sua vegni, corsi di aggiornamento per opera e il suo impegno ha fatto sì breve presentazione con l'invito ai giovani e agli specializzandi a interessarsi a queste patologie perché, anche se un po' messe da parte a favore delle "mode" specialistiche, possono senza dubbio dare grandi soddisfazioni a chi se ne occupa, ma particolarmente agli ammalati e ai loro genitori.

A. Memeo

Direttore Struttura Complessa Ortopedia e Traumatologia Pediatrica, Istituto Ortopedico G. Pini, Milano 